\title{
A simple protocol for the michael addition of indoles with electron deficient olefins catalysed by TBAHS in aqueous media and their broad spectrum antibacterial activity
}

\author{
M DAMODIRAN, ${ }^{a}$ R SENTHIL KUMAR, ${ }^{a}$ P M SIVAKUMAR, ${ }^{b}$ MUKESH DOBLE ${ }^{\mathrm{b}}$ and \\ PARAMASIVAN T PERUMAL ${ }^{\mathrm{a} *}$ \\ ${ }^{a}$ Organic Chemistry Division, Central Leather Research Institute, Adyar, Chennai 600020 \\ ${ }^{\mathrm{b}}$ Department of Biotechnology, Indian institute of Technology Madras, Adyar, Chennai 600036 \\ e-mail: ptperumal@gmail.com
}

MS received 18 July 2008; revised 28 September 2008

\begin{abstract}
Tetrabutylammonium hydrogen sulfate catalysed addition of indoles to electron deficient olefins in water generated the corresponding Michael adducts in good to excellent yield. The Michael addition of indole occurred regioselectively at position 3 and the $N$-alkylated products have not been observed. The synthesized compounds were tested for their antibacterial activity against four microorganisms namely, $E$. coli NCIM 2931, S. aureus NCIM 5021, P. vulgaris NCIM 2813, P. aeruginosa NCIM 5029 by micro dilution method. These compounds showed MIC (Minimum Inhibitory Concentration) values in the range of $0 \cdot 16-2 \cdot 67 \mu \mathrm{M}$.
\end{abstract}

Keywords. Michael addition; indole; water; antibacterial activity; tetrabutylammonium hydrogen sulfate.

\section{Introduction}

Organic reactions in water without the use of any harmful organic solvents are of great current interest, because water is an easily available, economical, safe, and environmentally benign solvent. Recently, some attempts to achieve organic reactions in aqueous media have been performed, and conesquently some successful examples have appeared in the literature. ${ }^{1,2}$ The development of an efficient and convenient synthetic methodology to accomplish $\mathrm{C}-\mathrm{C}$ bond formation in water is of paramount significance because of heightened importance in green chemistry. In this context, in recent years, much attention has been focused on Lewis acid catalysed organic reactions in water.

The 3-substituted indole nucleus is prevalent in numerous natural products and is extremely important in medicinal chemistry. ${ }^{3}$ The development of synthetic methods leading to indole derivatives has attracted much attention in organic synthesis because of their biological activities. ${ }^{2,3}$ The conjugate addition of indoles to $\alpha, \beta$-unsaturated ketones constitutes a key reaction in the total synthesis of com-

\footnotetext{
*For correspondence
}

plex natural products such as hapalindole. ${ }^{4-6}$ The hapalindole alkaloids were isolated from the bluegreen algae Hapalosiphon fontinalis. They exhibit potent antibacterial and antimycotic activities and have attracted the interest of both synthetic and pharmaceutical chemist. ${ }^{7}$ Various indole derivatives are components of drugs and are commonly found in molecules of pharmaceutical interest in a variety of therapeutic areas. Generally, 3-substituted indoles exhibit numerous biological activities. Therefore, a variety of methods have been reported for the preparation of this class of compounds. In animals, serotonin (5-hydroxytryptamine) is a crucial neurotransmitter in the central nervous system. The potent physiological properties of these indole derivatives led to vast research of their use as medicines in the field of pharmaceutical chemistry. Among the successful examples as drugs are sumatriptan, which is used in the treatment of migraine headaches, and pindolol, one of the $\beta$-adrenergic blockers, almotriptan and avitriptan acts as a $5-\mathrm{HT}_{1 \mathrm{D}}$ receptor agonists. 3-acetyl indoles are drug fragments commonly found in molecules of pharmaceutical interest in a variety of therapeutic areas. Interesting examples include the analgesic, pravodoline and the antiemetic, Ramosetron. ${ }^{8}$ 
The simple and direct method for the synthesis of 3 -alkylated indoles involves the conjugate addition of indoles to $\alpha, \beta$-unsaturated compounds in the presence of either protic or Lewis acids. The Lewis acids employed include $\mathrm{InCl}_{3},{ }^{9} \mathrm{Zr}(\mathrm{OTf})_{2},{ }^{10} \mathrm{SmI}_{3},{ }^{11}$ $\mathrm{CeCl}_{3} \cdot 7 \mathrm{H}_{2} \mathrm{O}-\mathrm{NaI} .{ }^{12}$ However, most of these Lewis acids are moisture sensitive and hence difficult to handle. Also their cost is of concern especially when considering the scale up of the reaction. Recently studies have been carried out to explore tetrabutylammonium hydrogen sulfate (TBAHS) for its catalytic activity in organic synthesis. For example, TBAHS has been used as an efficient catalyst for acid catalysed reaction and as a phase transfer catalyst in the synthesis of aryl vinyl ether, ${ }^{13}$ glycosylation of hydroxamic acid, ${ }^{14}$ synthesis of dihydropyridines. ${ }^{15,16}$ Moreover, some important organic transformations, like selective oxidation of benzyl alcohols, ${ }^{17}$ have been performed successfully in the presence of TBAHS. TBAHS, an acidic catalyst could prove ideal for synthetic applications in aqueous medium provided, the catalyst exhibits high selectivity. In continuation of our earlier work, ${ }^{18}$ the Michael addition of indoles to olefins in water catalysed by TBAHS was explored. Thus the treatment of indoles with electron deficient olefins in the presence of catalytic amount of TBAHS leads to the formation of 3-alkylated indoles in good to excellent yield.

TBAHS is a readily available and economically feasible solid acid catalyst that offers several advantages. TBAHS, being acidic in nature was chosen as catalyst. Organic reactions that exploit TBAHS as catalyst in water could prove ideal for synthetic organic chemistry applications, provided the catalyst show high catalytic activity in water.

\section{Experimental}

Melting points are uncorrected. IR spectra were recorded on a Perkin Elmer FT-IR spectrophotometer. ${ }^{1} \mathrm{H}$ and ${ }^{13} \mathrm{C}$ NMR spectra were recorded in $\mathrm{CDCl}_{3}$ using TMS as an internal standard on a JEOL spectrometer at $500 \mathrm{MHz}$ and $125 \mathrm{MHz}$, respectively. Mass spectra were recorded on a Thermo Finnigan LCQ Advantage MAX 6000 ESI spectrometer. Elemental analyses were recorded using a Thermo Finnigan FLASH EA $1112 \mathrm{CHN}$ analyzer. Column chromatography was performed on silica gel (100200 mesh, SRL, India). Analytical TLC was performed on pre-coated plastic sheets of silica gel G/UV-254 of $0.2 \mathrm{~mm}$ thickness (Merck, Germany).

\subsection{Experimental procedure for compound $3 \boldsymbol{b}$}

A mixture of indole $(4.2 \mathrm{mmol}), \beta$-nitrostyrene $(4.2 \mathrm{mmol})$ and Tetra-n-butylammonium hydrogen sulfate $(50 \mathrm{~mol} \%)$ was stirred in water $(15 \mathrm{~mL})$ at room temperature until completion of the reaction as evidenced by TLC analysis. The reaction mixture was extracted with ethyl acetate $(2 \times 30 \mathrm{~mL})$ and the organic layers were separated carefully from the aqueous layer. The combined organic layers were dried over anhydrous $\mathrm{Na}_{2} \mathrm{SO}_{4}$ by which the water present after the extraction can be removed and further, the organic layer is concentrated in vacuum. The crude was purified by column chromatography on silica gel (Merck, 100-200 mesh, ethyl acetatepetroleum ether $(10: 90)$.

2.1a 4-1H-Indol-3-yl)butan-2-one (3a): Colourless solid; m.p. $87-89^{\circ} \mathrm{C}$; IR $v_{\max }(\mathrm{KBr}) 3033,1689$, $1588,1357 \mathrm{~cm}^{-1} ;{ }^{1} \mathrm{H}$ NMR $\left(500 \mathrm{MHz}, \mathrm{CDCl}_{3}\right) \delta$ $8.10(s, 1 \mathrm{H}), 7.60(d, 1 \mathrm{H}, J=7.4 \mathrm{~Hz}), 7.34(t, 1 \mathrm{H}$, $J=3.4 \mathrm{~Hz}), 7 \cdot 19-7.26(m, 1 \mathrm{H}), 7 \cdot 13-7 \cdot 13(m, 1 \mathrm{H})$, $6.95(d, 1 \mathrm{H}, J=2.3 \mathrm{~Hz}), 3.07(t, 2 \mathrm{H}, J=2.85 \mathrm{~Hz})$ $2.85(t, 2 \mathrm{H}, J=3.4 \mathrm{~Hz}), 2.15(s, 3 \mathrm{H}) ;{ }^{13} \mathrm{C}$ NMR $\left(125 \mathrm{MHz}, \mathrm{CDCl}_{3}\right) \delta 209 \cdot 0,138 \cdot 4,127 \cdot 2,122 \cdot 1$, $121 \cdot 6,119 \cdot 3,118 \cdot 7,115 \cdot 1,111 \cdot 3,44 \cdot 2,30 \cdot 1,19 \cdot 4$; MS (EI) $m / z \quad 187 \quad\left(\mathbf{M}^{+}+1\right)$. Anal. Calcd. for $\mathrm{C}_{12} \mathrm{H}_{13} \mathrm{NO}$ : C 76.98, H 7.00, N 7.48. Found C 76.87, $\mathrm{H} 7 \cdot 11, \mathrm{~N} 7 \cdot 43$.

$2.1 \mathrm{~b} \quad 3-(2-N i t r o-1-p h e n y l e t h y l)-1 H$-indole $\quad(3 \boldsymbol{b})$ : Pink solid; m.p. $91-93^{\circ} \mathrm{C}$; IR $v_{\max }(\mathrm{KBr}) 3400,1536$, 1424, 1376; ${ }^{1} \mathrm{H}$ NMR $\left(500 \mathrm{MHz}, \mathrm{CDCl}_{3}\right) \delta 8 \cdot 14(s$, $1 \mathrm{H}), 7.46(d, 1 \mathrm{H}, J=8.4 \mathrm{~Hz}), 7.31-7.34(m, 5 \mathrm{H})$, $7.25-7.33(m, 1 \mathrm{H}), 7.20(t, 1 \mathrm{H}, J=7.6 \mathrm{~Hz}), 7.09(t$, $1 \mathrm{H}, J=8.4 \mathrm{~Hz}), 6.98(d, 1 \mathrm{H}, J=2.3 \mathrm{~Hz}), 5.19(t$, $1 \mathrm{H}, J=7.6 \mathrm{~Hz}), 5.05(d d, 1 \mathrm{H}, J=7.6 \mathrm{~Hz}, 12.2 \mathrm{~Hz})$, $4.94(d d, \quad 1 \mathrm{H}, J=8.4 \mathrm{~Hz}, 12.2 \mathrm{~Hz}) ;{ }^{13} \mathrm{C}$ NMR $\left(125 \mathrm{MHz}, \mathrm{CDCl}_{3}\right) \delta 139 \cdot 3,136 \cdot 5,129 \cdot 0,127 \cdot 8$, $126 \cdot 2,122 \cdot 7,121 \cdot 7,120 \cdot 0,119 \cdot 0,114 \cdot 4,111 \cdot 5$, 79.6, 41.6; MS (EI) $m / z 267\left(\mathbf{M}^{+}+1\right)$. Anal. Calcd. for $\mathrm{C}_{16} \mathrm{H}_{14} \mathrm{~N}_{2} \mathrm{O}_{2}$ : C $72 \cdot 17, \mathrm{H} 5 \cdot 30, \mathrm{~N}$ 10.52. Found $\mathrm{C}$ $72 \cdot 25, \mathrm{H} 5 \cdot 27, \mathrm{~N} 10 \cdot 48$.

\section{1c 3-[1-(4-Methoxyphenyl)-2-nitroethyl]- $1 \mathrm{H}$ -}

indole (3c): Colourless; m.p. $144-146^{\circ} \mathrm{C}$; IR $v_{\max }$ (KBr) 3380, 1547, 1422, 1376; ${ }^{1} \mathrm{H}$ NMR $(500 \mathrm{MHz}$, $\left.\mathrm{CDCl}_{3}\right) \delta 9.55(s, 1 \mathrm{H}), 7.31(d, 1 \mathrm{H}, J=7.6 \mathrm{~Hz}), 7.27$ $(d, 1 \mathrm{H}, J=7.6 \mathrm{~Hz}), 7.14(d, 2 \mathrm{H}, J=9.2 \mathrm{~Hz}), 7.05(t$, $1 \mathrm{H}, J=7.6 \mathrm{~Hz}), 6.91-6.94(m, 2 \mathrm{H}), 6.73(d, 2 \mathrm{H}$, $J=8.4 \mathrm{~Hz}), 5.00(t, 1 \mathrm{H}, J=8.5 \mathrm{~Hz}), 4.92(d d, 1 \mathrm{H}$, $J=7.6 \mathrm{~Hz}, \quad 12.2 \mathrm{~Hz}), \quad 4.80 \quad(d d, 1 \mathrm{H}, J=8.4 \mathrm{~Hz}$, 
$12.2 \mathrm{~Hz}), \quad 3.65 \quad(s, \quad 3 \mathrm{H}) ;{ }^{13} \mathrm{C} \quad \mathrm{NMR} \quad(125 \mathrm{MHz}$, $\left.\mathrm{CDCl}_{3}\right): \delta 158 \cdot 7,136 \cdot 7,131 \cdot 5,128 \cdot 8,126 \cdot 8,122 \cdot 1$, $121 \cdot 9,118 \cdot 7,114 \cdot 2,113 \cdot 8,111 \cdot 7,79 \cdot 8,55 \cdot 2,41 \cdot 6$; MS (EI) $m / z \quad 297 \quad\left(\mathbf{M}^{+}+1\right)$. Anal. Calcd. for $\mathrm{C}_{17} \mathrm{H}_{16} \mathrm{~N}_{2} \mathrm{O}_{3}$ : C 68.91, H 5.44, N 9.45. Found $\mathrm{C}$ $68 \cdot 86, \mathrm{H} 5 \cdot 47, \mathrm{~N} 9 \cdot 48$.

\section{1d 5-Bromo-3-(2-nitro-1-phenylethyl)-1H-indole} (3d): Colourless solid; m.p. $73-75^{\circ} \mathrm{C}$; IR $v_{\max }(\mathrm{KBr})$ $3431,1549,1454,1375 ;{ }^{1} \mathrm{H}$ NMR $(500 \mathrm{MHz}$, $\left.\mathrm{CDCl}_{3}\right) \delta 8.17(s, 1 \mathrm{H}), 7.55(d, 1 \mathrm{H}, J=6.3 \mathrm{~Hz})$, $7 \cdot 25-7.32(m, 6 \mathrm{H}), 7 \cdot 17-7.21(m, 1 \mathrm{H}), 7.03(t, 1 \mathrm{H}$, $J=5 \cdot 1 \mathrm{~Hz}), 5 \cdot 11(t, 1 \mathrm{H}, J=7 \cdot 6 \mathrm{~Hz}), 5 \cdot 00-5.04(m$, 1H) $4.90-4.94 \quad(m, 1 \mathrm{H}) ;{ }^{13} \mathrm{C}$ NMR $(125 \mathrm{MHz}$, $\left.\mathrm{CDCl}_{3}\right) \delta 138 \cdot 8,135 \cdot 1,129 \cdot 1,127 \cdot 9,127 \cdot 7,125 \cdot 7$, $122 \cdot 8,121 \cdot 5,120 \cdot 0,119 \cdot 0,114 \cdot 0,113 \cdot 3,79 \cdot 5,41 \cdot 4$; MS (EI) $m / z 345\left(\mathbf{M}^{+}+1\right), 347\left(\mathbf{M}^{+}+2\right)$. Anal. Calcd. for $\mathrm{C}_{16} \mathrm{H}_{13} \mathrm{BrN}_{2} \mathrm{O}_{2}$ : C 55.67, H 3.80, N 8.12. Found C 55.72, H 3.82, N 8.19.

2.1e 5-Bromo-methoxyphenyl)-2-nitroethyl]-1Hindole (3e): Pale orange solid; m.p. $146-148^{\circ} \mathrm{C}$; IR $v_{\max }(\mathrm{KBr}) \quad 3388,1545,1457,1378 ;{ }^{1} \mathrm{H}$ NMR $\left(500 \mathrm{MHz}, \mathrm{CDCl}_{3}\right) \delta 10 \cdot 19(s, 1 \mathrm{H}), 7 \cdot 32(s, 1 \mathrm{H})$, $7.08(d, 1 \mathrm{H}, J=8.4 \mathrm{~Hz}), 7.02-7.04(m, 3 \mathrm{H}), 6.91(d$, $1 \mathrm{H}, J=2.3), 6.65(d, 2 \mathrm{H}, J=8.4 \mathrm{~Hz}), 4.81-4.85(m$, 2H), 4.69-4.7 (m, 1H), 3.54-3.58 $(\mathrm{s}, 3 \mathrm{H}),{ }^{13} \mathrm{C}$ NMR $\left(125 \mathrm{MHz}, \mathrm{CDCl}_{3}\right) \delta 162 \cdot 8,158.8,135.4,131 \cdot 1$, $128 \cdot 7,127 \cdot 8,124 \cdot 7,123 \cdot 2,121 \cdot 0,114 \cdot 2,113 \cdot 3$, 113.2, 79.7, 55.2; MS (EI) $m / z 376\left(\mathrm{M}^{+}+1\right), 378$ $\left(\mathrm{M}^{+}+2\right)$. Anal. Calcd. for $\mathrm{C}_{17} \mathrm{H}_{15} \mathrm{BrN}_{2} \mathrm{O}_{3}$ : C 54.42, $\mathrm{H} 4 \cdot 03$, N 7.47. Found C 54.38, H 4.15, N 7.42.

2.1f 2-Methyl-3-(2-nitro-1-phenylethyl)-1H-indole (3f): Pale pink solid; m.p. $91-93^{\circ} \mathrm{C}$; IR $v_{\max }(\mathrm{KBr})$ $3383,1548,1458,1375 ;{ }^{1} \mathrm{H}$ NMR $\left(500 \mathrm{MHz}, \mathrm{CDCl}_{3}\right.$ ) $\delta 7.87(s, 1 \mathrm{H}), 7.38(t, 1 \mathrm{H}, J=8.0 \mathrm{~Hz}), 7 \cdot 26-7.40$ $(m, 6 \mathrm{H}), 7.11(d, 1 \mathrm{H}, J=7.45 \mathrm{~Hz}), 7.03(d, 1 \mathrm{H}$, $J=7.4 \mathrm{~Hz}), 5 \cdot 11-5.22(m, 3 \mathrm{H}), 2.34(s, 3 \mathrm{H}) ;{ }^{13} \mathrm{C}$ NMR $\left(125 \mathrm{MHz}, \mathrm{CDCl}_{3}\right): \delta 139.6,136 \cdot 4,133.0$, $128.9,127 \cdot 2,122 \cdot 1,121 \cdot 6,119 \cdot 3,118 \cdot 7,115 \cdot 1$, $111 \cdot 3,44 \cdot 2,30 \cdot 1,19 \cdot 4$; $\mathrm{MS}(\mathrm{EI}) \mathrm{m} / z 281\left(\mathrm{M}^{+}+1\right)$. Anal. Calcd. for $\mathrm{C}_{17} \mathrm{H}_{16} \mathrm{~N}_{2} \mathrm{O}_{2}$ : C 72.84, H 5.75, N 9.99 Found C 72.79, H 5.69, N 9.94.

\section{$2.2 \mathrm{~g}$ 5-Methoxy-3-(2-nitro-1-phenylethyl)-1H-}

indole (3g): Pale pink solid; m.p. $105-107^{\circ} \mathrm{C}$; IR $v_{\max }(\mathrm{KBr}) \quad 3446,1548,1481,1378 ;{ }^{1} \mathrm{H}$ NMR $\left(500 \mathrm{MHz}, \mathrm{CDCl}_{3}\right) \delta 8.03(s, 1 \mathrm{H}), 7.30-7.33(\mathrm{~m}$, $4 \mathrm{H}), 7.25-7.28(m, 1 \mathrm{H}), 7.21(d, 1 \mathrm{H}, J=9.2 \mathrm{~Hz})$, $6 \cdot 96(d, 1 \mathrm{H}, J=2.3 \mathrm{~Hz}), 6 \cdot 85-6 \cdot 87(m, 2 \mathrm{H}), 5 \cdot 14(t$,
$1 \mathrm{H}, J=7.6 \mathrm{~Hz}), 5.03(d d, 1 \mathrm{H}, J=7.6 \mathrm{~Hz}, 12 \cdot 2 \mathrm{~Hz})$, $4.92(d d, 1 \mathrm{H}, J=8.4 \mathrm{~Hz}, 12.2 \mathrm{~Hz}), 3.77(s, 3 \mathrm{H}) ;{ }^{13} \mathrm{C}$ NMR $\left(125 \mathrm{MHz}, \mathrm{CDCl}_{3}\right): \delta 154 \cdot 2,139.2,131.7$, $129 \cdot 0,127 \cdot 8,127 \cdot 6,126 \cdot 6,122 \cdot 4,114 \cdot 0,112 \cdot 7$, $112 \cdot 2,100 \cdot 9,79 \cdot 6,55 \cdot 9,41 \cdot 6$; MS (EI) $\mathrm{m} / z 297$ $\left(\mathrm{M}^{+}+1\right)$. Anal. Calcd. for $\mathrm{C}_{17} \mathrm{H}_{16} \mathrm{~N}_{2} \mathrm{O}_{3}$ : C 68.91, H 5.44, N 9.45. Found C 68.80, H 5.49.

2.1h 1-Methyl-3-(2-nitro-1-phenylethyl)-1H-indole (3h): Pink solid; m.p. $83-85^{\circ} \mathrm{C}$. IR $v_{\max }(\mathrm{KBr}) 3060$, 2942, 1424, 1376; ${ }^{1} \mathrm{H}$ NMR $\left(500 \mathrm{MHz}, \mathrm{CDCl}_{3}\right) \delta$ $7.47(d, 1 \mathrm{H}, J=7.6 \mathrm{~Hz}), 7.33-7.36(m, 3 \mathrm{H}), 7.32(d$, $1 \mathrm{H}, J=3.05 \mathrm{~Hz}), 7.31(s, 1 \mathrm{H}), 7.26(m, 2 \mathrm{H}), 7.09(t$, $1 \mathrm{H}, J=6.9 \mathrm{~Hz}), 6.87 \quad(s, 1 \mathrm{H}), 5.19 \quad(t, 1 \mathrm{H}$, $J=8.4 \mathrm{~Hz}), 5.05(d d, 1 \mathrm{H}, J=7.6 \mathrm{~Hz}, 12.2 \mathrm{~Hz}), 4.94$ $(d d, 1 \mathrm{H}, J=8.4 \mathrm{~Hz}, 12.2 \mathrm{~Hz}), 3.73(s, 3 \mathrm{H}) ;{ }^{13} \mathrm{C}$ NMR $\left(125 \mathrm{MHz}, \mathrm{CDCl}_{3}\right) \delta 139.4,137.3,129.0$, $127 \cdot 85,126 \cdot 6,122 \cdot 3,119 \cdot 5,119 \cdot 0,112 \cdot 8,109 \cdot 6$, 79.6, 41.6, 32.9; MS (EI) $m / z 281\left(\mathbf{M}^{+}+1\right)$. Anal. Calcd. for $\mathrm{C}_{17} \mathrm{H}_{16} \mathrm{~N}_{2} \mathrm{O}_{2}$ : C 72.84, H 5.75, N 9.99. Found C 72.81, H 5.80, N 9.96.

2.1i 3-(1H-Indole-3-yl)-1,3-diphenylpropan-1-one (3i): Pink solid; m.p. $106-108^{\circ} \mathrm{C}$; IR $v_{\max }(\mathrm{KBr})$ $3404,1675,1457,1336 ;{ }^{1} \mathrm{H}$ NMR $(500 \mathrm{MHz}$, $\left.\mathrm{CDCl}_{3}\right) \delta 8.08(s, 1 \mathrm{H}), 7.94(d, 2 \mathrm{H}, J=7.65 \mathrm{~Hz})$, $7.54(t, 1 \mathrm{H}, J=6.90 \mathrm{~Hz}), 7.43(t, 3 \mathrm{H}, J=7.6 \mathrm{~Hz})$, $7.35(d, 2 \mathrm{H}, J=7.6 \mathrm{~Hz}), 7.31(d, 1 \mathrm{H}, J=7.6 \mathrm{~Hz})$, $7.24-7.27(m, 2 \mathrm{H}), 7.15(q, 2 \mathrm{H}, J=7.6 \mathrm{~Hz}), 7.02(t$, $1 \mathrm{H}, J=7.6 \mathrm{~Hz}), 6.97 \quad(s, 1 \mathrm{H}), \quad 5.08 \quad(d, 1 \mathrm{H}$, $J=6.9 \mathrm{~Hz}), 5.82(d d, 1 \mathrm{H}, J=6.8 \mathrm{~Hz}, 16.8 \mathrm{~Hz}), 5.73$ $(d d, 1 \mathrm{H}, J=7.6 \mathrm{~Hz}, 16.8 \mathrm{~Hz}) ;{ }^{13} \mathrm{C}$ NMR $(125 \mathrm{MHz}$, $\left.\mathrm{CDCl}_{3}\right) \delta 198 \cdot 7,163 \cdot 6,144 \cdot 3,137 \cdot 1,136 \cdot 7,133 \cdot 1$, $128 \cdot 7,128 \cdot 5,128 \cdot 2,127 \cdot 9,126 \cdot 7,126 \cdot 4,122 \cdot 2$, $121.5,119 \cdot 6,119 \cdot 5,119 \cdot 3,111.2,38.3,45 \cdot 3$; MS (EI) $m / z 326\left(\mathbf{M}^{+}+1\right)$. Anal. Calcd. for $\mathrm{C}_{23} \mathrm{H}_{19} \mathrm{NO}$ : C 84.89, H $5.88, \mathrm{~N} 4 \cdot 30$. Found C $84.84, \mathrm{H} 5.92$, N $4 \cdot 27$.

$2.1 \mathrm{j} \quad 1-(4-B r o m o p h e n y l)-3-1 H$-indole-3-yl)-3-diphenylpropan-1-one (3j): Colourless; m.p. 174$176^{\circ} \mathrm{C}$; IR $v_{\max }(\mathrm{KBr}) 3444,1679,1580,1457 ;{ }^{1} \mathrm{H}$ $\operatorname{NMR}\left(500 \mathrm{MHz}, \mathrm{CDCl}_{3}\right) \delta 9.88(s, 1 \mathrm{H}), 7.67(d, 2 \mathrm{H}$, $J=8.4 \mathrm{~Hz}), 7.46(d, 2 \mathrm{H}, J=8.4 \mathrm{~Hz}), 7.28(d, 2 \mathrm{H}, J=$ $8.4 \mathrm{~Hz}), 7.22(d, 2 \mathrm{H}, J=6.9 \mathrm{~Hz}), 7.12(t, 2 \mathrm{H}, J=$ $7.6 \mathrm{~Hz}), 7.02(t, 1 \mathrm{H}, J=7.6 \mathrm{~Hz}), 6.97(t, 1 \mathrm{H}$, $J=7.6 \mathrm{~Hz}), 6.92(d, 1 \mathrm{H}, J=2.3 \mathrm{~Hz}), 6.83(t, 1 \mathrm{H}, J=$ $7.6 \mathrm{~Hz}), 4.87(t, 1 \mathrm{H}, J=7.6 \mathrm{~Hz}), 3.67(d d, 1 \mathrm{H}$, $J=6.8 \mathrm{~Hz}, 16.8 \mathrm{~Hz}), 3.57(d d, 1 \mathrm{H}, J=8.4 \mathrm{~Hz}$, $16.8 \mathrm{~Hz}) ;{ }^{13} \mathrm{C}$ NMR $\left(125 \mathrm{MHz}, \mathrm{CDCl}_{3}\right) \delta 198 \cdot 7$, $163.6,144.3,136.8,135.8,131.8,129.7,128.4$, 
$128 \cdot 0,127 \cdot 8,126 \cdot 5,126 \cdot 2,121 \cdot 7,121 \cdot 6,119 \cdot 1$, $118 \cdot 8,118 \cdot 1,111 \cdot 5,45 \cdot 1,40 \cdot 1$; MS (EI) $\mathrm{m} / z \quad 404$ $\left(\mathrm{M}^{+}+1\right), 406\left(\mathrm{M}^{+}+2\right)$. Anal. Calcd. for $\mathrm{C}_{23} \mathrm{H}_{18} \mathrm{BrNO}$ : C 68.33, H 4.49, N 3.46 Found C 68.37, H 4.43, N $3 \cdot 518$.

$2.1 \mathrm{k} \quad 3-[4-(D i m e t h y l a m i n o p h e n y l)-3-1 H$-indole-3yl]-1-phenylpropan-1-one (3k): Pale pink solid; m.p. 167- $169^{\circ} \mathrm{C}$; IR $v_{\max }(\mathrm{KBr}) 3424,1674,1520,1336$; ${ }^{1} \mathrm{H}$ NMR $\left(500 \mathrm{MHz}, \mathrm{CDCl}_{3}\right) \delta 10.75(s, 1 \mathrm{H}), 7.95$ $(d, 1 \mathrm{H}, J=7.6 \mathrm{~Hz}), 7.58(t, 1 \mathrm{H}, J=7.6 \mathrm{~Hz}), 7.47(t$, $1 \mathrm{H}, J=7.6 \mathrm{~Hz}), 7.34 \quad(d, 1 \mathrm{H}, J=7.6 \mathrm{~Hz}), 7.24$ $(t, 1 \mathrm{H}, J=9.1 \mathrm{~Hz}), 7.13(d, 1 \mathrm{H}, J=8.4 \mathrm{~Hz}), 6.97$ $(t, 4 \mathrm{H}, J=7.6 \mathrm{~Hz}), 6.83(t, 3 \mathrm{H}, J=7.6 \mathrm{~Hz}), 6.54(d$, $1 \mathrm{H}, J=8.4 \mathrm{~Hz}), 4.72(t, 1 \mathrm{H}, J=6.9 \mathrm{~Hz}), 3.79(d d$, $1 \mathrm{H}, J=6.8 \mathrm{~Hz}, 16.8 \mathrm{~Hz}), 3.69(d d, 1 \mathrm{H}, J=7.6 \mathrm{~Hz}$, $16.8 \mathrm{~Hz}), \quad 3.32 \quad(s, \quad 6 \mathrm{H}) ;{ }^{13} \mathrm{C}$ NMR $(125 \mathrm{MHz}$, $\left.\mathrm{CDCl}_{3}\right): \delta 199 \cdot 1,149 \cdot 2,137 \cdot 4,136 \cdot 9,133.5,133 \cdot 4$, $129.2,128 \cdot 6,128.5,126 \cdot 9,122 \cdot 1,121 \cdot 3,119 \cdot 3$, $119 \cdot 2,118 \cdot 6,112 \cdot 9,111 \cdot 7,45 \cdot 0,40 \cdot 8$; MS (EI) $m / z$ $369\left(\mathrm{M}^{+}+1\right)$. Anal. Calcd. for $\mathrm{C}_{25} \mathrm{H}_{24} \mathrm{~N}_{2} \mathrm{O}$ : C 81.49, H 6.56, N 7.60. Found C 81.42, H 6.59, N 7.57.

2.11 1-(4-Chlorophenyl)-3-(1H-indole-3-yl)-3-phenylpropan-1-one (3l): Colourless; m.p. $92-94^{\circ} \mathrm{C}$; IR $v_{\max }(\mathrm{KBr}) \quad 3444,1679,1585,1455 ;{ }^{1} \mathrm{H}$ NMR $\left(500 \mathrm{MHz}, \mathrm{CDCl}_{3}\right) \delta 7.98(s, 1 \mathrm{H}), 7.84(d, 2 \mathrm{H}$, $J=8.4 \mathrm{~Hz}), \quad 7.43 \quad(d, \quad 1 \mathrm{H}, J=7.6 \mathrm{~Hz}), 7.38 \quad(d$, $2 \mathrm{H}, J=8.4 \mathrm{~Hz}), 7.33(t, 3 \mathrm{H}, J=6.8 \mathrm{~Hz}), 7.25(t, 2 \mathrm{H}$, $J=7.5 \mathrm{~Hz}), \quad 7.13-7.17 \quad(m, 2 \mathrm{H}), \quad 7.01 \quad(t, \quad 1 \mathrm{H}$, $J=8.4 \mathrm{~Hz}), 6.97(s, 1 \mathrm{H}), 5.03(t, 1 \mathrm{H}, J=7.6 \mathrm{~Hz})$, $3.77(d d, 1 \mathrm{H}, J=6.9 \mathrm{~Hz}, 16.8 \mathrm{~Hz}), 3.67(d d, 1 \mathrm{H}$, $J=7.6 \mathrm{~Hz}, 16.8 \mathrm{~Hz}) ;{ }^{13} \mathrm{C}$ NMR $\left(125 \mathrm{MHz}, \mathrm{CDCl}_{3}\right)$ : $\delta 197.5,144 \cdot 0,139.5,136 \cdot 7,135.5,129.6,128.5$, $127 \cdot 8,126 \cdot 6,126 \cdot 4,121.4,119.5,119.2,111 \cdot 2$, 40.2, 38.4; MS (EI) $m / z 359\left(\mathbf{M}^{+}\right)$. Anal. Calcd. for $\mathrm{C}_{23} \mathrm{H}_{18} \mathrm{ClNO}$ : C 76.77, $\mathrm{H} 5.04, \mathrm{~N} 3.89$ Found C 76.81, H 5.11, N 3.84. requires \%. MS $\mathrm{m} / \mathrm{z} 360$ $\left(\mathrm{M}^{+}+1\right), 362\left(\mathrm{M}^{+}+2\right)$.

$2.1 \mathrm{~m}$ 1-(4-Bromophenyl)-3-(2-methyl-1H-indole3-yl)-3-phenylpropan-1-one (3m): Brown solid; IR $v_{\max }(\mathrm{KBr}) \quad 3402,2915,1679,1583 ;{ }^{1} \mathrm{H}$ NMR $\left(500 \mathrm{MHz}, \mathrm{CDCl}_{3}\right) \delta 7.75(s, 1 \mathrm{H}), 7.69(d, 2 \mathrm{H}$, $J=8.4 \mathrm{~Hz}), 7.48(d, 2 \mathrm{H}, J=8.4 \mathrm{~Hz}), 7.44(d, 1 \mathrm{H}$, $J=8.4 \mathrm{~Hz}), 7.34(d, 2 \mathrm{H}, J=7.6 \mathrm{~Hz}), 7.20-7.27(m$, $3 \mathrm{H}), 7.15(t, 1 \mathrm{H}, J=7.65 \mathrm{~Hz}), 7.06(t, 1 \mathrm{H}, J=6.9 \mathrm{~Hz})$, $6.99(t, 1 \mathrm{H}, J=7.6 \mathrm{~Hz}), 5.05(t, 1 \mathrm{H}, J=6.9 \mathrm{~Hz})$, $3.92(d d, 1 \mathrm{H}, J=8.4 \mathrm{~Hz}, 16.8 \mathrm{~Hz}), 3.81(d d, 1 \mathrm{H}$, $J=6.1 \mathrm{~Hz}, \quad 16.0 \mathrm{~Hz}), \quad 2.38 \quad(3, \quad 3 \mathrm{H}) ;{ }^{13} \mathrm{C}$ NMR $\left(125 \mathrm{MHz}, \mathrm{CDCl}_{3}\right) \delta 198 \cdot 3,144 \cdot 0,135 \cdot 9,135 \cdot 5$,
$131 \cdot 8, \quad 129 \cdot 6,128 \cdot 4,128 \cdot 1,127 \cdot 5,127 \cdot 4,126 \cdot 1$, $120 \cdot 9,119 \cdot 3,119 \cdot 1,113 \cdot 4,110 \cdot 5,43 \cdot 4,37 \cdot 0$ 12.2; MS (EI) $m / z 418\left(\mathbf{M}^{+}+1\right), 420\left(\mathbf{M}^{+}+1\right)$. Anal. Calcd. for $\mathrm{C}_{24} \mathrm{H}_{20} \mathrm{BrNO}$ : $\mathrm{C}$ 68.91, H 4.82, N 3.35. Found C 68.94, H 4.77, N 3.29.

2.1n 3-[4-(Dimethylamino)phenyl]-3-(2-methyl-1Hindole-3-yl)-1-phenylpropan-1-one (3n): Brown solid; m.p. $106-108^{\circ} \mathrm{C}$. IR $v_{\max }(\mathrm{KBr}) 3399,1680$, 1613,$1519 ;{ }^{1} \mathrm{H}$ NMR $\left(500 \mathrm{MHz}, \mathrm{CDCl}_{3}\right) \delta 7.87(d$, $2 \mathrm{H}, J=7.6 \mathrm{~Hz}), 7.77(s, 1 \mathrm{H}), 7.47-7.52(m, 2 \mathrm{H})$, $7.37(t, 2 \mathrm{H}, J=7.6 \mathrm{~Hz}), 7.23(d, 2 \mathrm{H}, J=8.4 \mathrm{~Hz}), 7.19$ $(d, 1 \mathrm{H}, J=7.6 \mathrm{~Hz}), 7.04(t, 1 \mathrm{H}, J=6.9 \mathrm{~Hz}), 7.01(t$, $1 \mathrm{H}, J=6.8 \mathrm{~Hz}), 6.66(d, 2 \mathrm{H}, J=8.4 \mathrm{~Hz}), 5.01(t$, $1 \mathrm{H}, J=6.9 \mathrm{~Hz}), 4.95(d d, 1 \mathrm{H}, J=7.6 \mathrm{~Hz}, 16.5 \mathrm{~Hz})$, $3.87(d d, 1 \mathrm{H}, J=6.8 \mathrm{~Hz}, 16.8 \mathrm{~Hz}), 2.88(s, 6 \mathrm{H})$, $2.35(s, 1 \mathrm{H}) ;{ }^{13} \mathrm{C}$ NMR $\left(125 \mathrm{MHz}, \mathrm{CDCl}_{3}\right) \delta 199.6$, $149 \cdot 0,137 \cdot 3,135 \cdot 6,132 \cdot 8,132 \cdot 5,131 \cdot 6,128 \cdot 5,128 \cdot 2$, $128 \cdot 1,127 \cdot 6,120 \cdot 6,119 \cdot 4,119 \cdot 1,114 \cdot 0,112 \cdot 9$, $110 \cdot 4,44 \cdot 0,40 \cdot 9,36 \cdot 0,12 \cdot 2$; MS (EI) $\mathrm{m} / z \quad 354$ $\left(\mathrm{M}^{+}+1\right)$. Anal. Calcd. for $\mathrm{C}_{26} \mathrm{H}_{26} \mathrm{~N}_{2} \mathrm{O}$ : C 81.64, $\mathrm{H}$ 6.85, N 7.32 Found C 81.71, H 6.80, N 7.28.

2.10 3-(5-Methoxy-1H-indole-3-yl)-1,3-diphenylpropan-1-one (3o): Colourless solid; m.p. 141$143^{\circ} \mathrm{C}$; IR $v_{\max }(\mathrm{KBr}) 3367,1678,1583,1484 ;{ }^{1} \mathrm{H}$ NMR $\left(500 \mathrm{MHz}, \mathrm{CDCl}_{3}\right) \delta 7.93(d, 2 \mathrm{H}, J=7.6 \mathrm{~Hz})$, $7.90(s, 1 \mathrm{H}), 7.54(t, 1 \mathrm{H}, J=7.6 \mathrm{~Hz}), 7.43(t, 2 \mathrm{H}$, $J=8.4 \mathrm{~Hz}), 7.35(d, 2 \mathrm{H}, J=6.8 \mathrm{~Hz}), 7.24-7.27(m$, $2 \mathrm{H}), 7 \cdot 15-7.19(m, 2 \mathrm{H}), 6.95(d, 1 \mathrm{H}, J=2.3 \mathrm{~Hz})$, $6.84(d, 1 \mathrm{H}, J=2.3 \mathrm{~Hz}), 6.79-6.81(m, 1 \mathrm{H}), 5.02(t$, $1 \mathrm{H}, J=6.8 \mathrm{~Hz}), 3.79(d d, 1 \mathrm{H}, J=6.1 \mathrm{~Hz}, 16.0 \mathrm{~Hz})$, 3.69-3.74 $(m, 4 \mathrm{H}) ;{ }^{13} \mathrm{C}$ NMR $\left(125 \mathrm{MHz}, \mathrm{CDCl}_{3}\right) \delta$ $198 \cdot 7,153 \cdot 8,144 \cdot 2,137 \cdot 2,133 \cdot 1,131 \cdot 8,128 \cdot 6$, $128 \cdot 5,128 \cdot 2,127 \cdot 9,127 \cdot 1,126 \cdot 4,122 \cdot 2,119 \cdot 1$, $112 \cdot 3,111 \cdot 8,101 \cdot 5,55 \cdot 9,45 \cdot 2,38 \cdot 2$; MS (EI) $\mathrm{m} / z$ $356\left(\mathrm{M}^{+}+1\right)$. Anal. Calcd. for $\mathrm{C}_{24} \mathrm{H}_{21} \mathrm{NO}_{2}$ : C 81.10, H 5.95, N 3.94 Found C 81.17, H 5.88, N 3.98 .

\subsection{Determination of the minimum inhibitory concentration (MIC)}

Antibacterial activity for 15, 3-alkylated indole derivatives were performed against four Gram $(+)$ ve and Gram (-) ve bacterial strains namely, E. coli NCIM 2931, $S$. aureus NCIM 5021, $P$. vulgaris NCIM 2813, $P$. aeruginosa NCIM 5029. The microdilution method recommended by NCCLS (National Committee for Clinical Laboratory Standrds) ${ }^{21}$ was followed with smaller modification of methodology reported by Sarker et $a l^{22}$ using 96 well plates. 
Other researchers have used resazurin assay for decades to find out the bacterial and yeast contamination in milk. ${ }^{23,24}$ Compounds were initially dissolved in $10 \%(\mathrm{v} / \mathrm{v})$ dimethylsulfoxide (DMSO) at a concentration of $10 \mathrm{mg} / \mathrm{ml}$ of stock. $100 \mu \mathrm{L}$ of the test compound was added into of the first row three wells (triplicates) which contained double the strength Mueller-Hinton broth. $100 \mu \mathrm{L}$ of single strength Mueller-Hinton broth was added into other wells. Serial dilution of the test compound was done using multichannel pipette in such a way that all the wells had $100 \mu \mathrm{L}$ of the test compound in serially decreasing concentration. $10 \mu \mathrm{L}$ of the bacterial culture $\left(5 \times 10^{6} \mathrm{cfu} / \mathrm{mL}\right)$ was added into each well to obtain a final concentration of $5 \times 10^{6} \mathrm{cfu} / \mathrm{mL}$. One entire column had antibiotics as a positive control (Norfloxacin/Erythromycin). A column with all the solutions except the test compound, and a column with all the solutions except the bacterial culture acted as controls. The plates were prepared in triplicate and incubated for $18 \mathrm{~h}$ at $37^{\circ} \mathrm{C}$ then $10 \mu \mathrm{L}$ of $0.01 \%$ resazurin solution was added and incubated for $2 \mathrm{~h}$. The colour change was assessed visually and the highest dilution remained blue (inhibition of growth) indicating minimum inhibitory concentration. Growth of organism changed the colour from blue to pink. The principle for the colour reaction is given below:

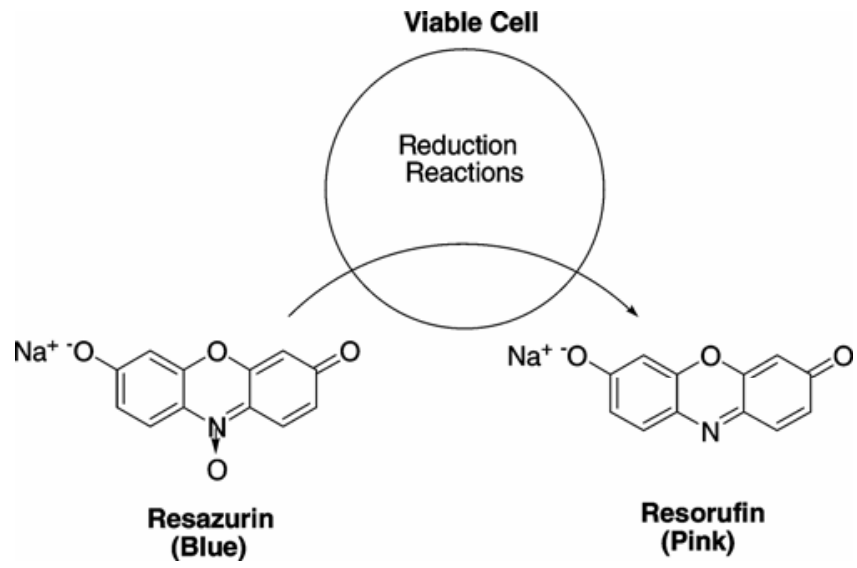

\section{Results and discussion}

\subsection{Synthesis}

In our study, the addition of indoles to electron deficient olefines, reaction of indole with nitrotyrene in presence of 0.5 equivalent of tetrautylammonium hydrogen sulfate at room temperature gives the corresponding 3-alkylated indole in $80 \%$ yield (scheme
1 , entry 1). We have carried out the addition of a variety of indoles with nitrostyrenes in good to excellent yield (75-90\%) (table 1, entries 2-8).

It has been observed that the electronic properties of the aromatic ring have an effect on the rate of this Michael reaction. The rate is accelerated by electron donating groups present on the indole nucleus. Indole substrate bearing an electron-withdrawing group afford the adduct in less yield compared to other indoles. With respect to the regioselectivity, it is observed that all the Michael acceptors gave exclusively the 1,4-adduct without any trace of 1,2adduct. Although some Michael adducts were obtained in good yield, the reaction times varied according to the nature of substitution pattern on the phenyl ring of nitrostyrene. The electron donating groups present in the phenyl ring of nitrostyrene led to formation of products with longer reaction times.

In order to extend the scope of this methodology, various 1,2-disubstituted vinyl ketones (chalcones) were used as Michael acceptor and the results are tabulated (table 1). In this case, however, reaction proceeds to completion at slightly elevated tempeature $\left(70^{\circ} \mathrm{C}\right)$. Chalcones having electron donating groups in the styryl ring gave the Michael adducts in shorter reaction time and good yield (entries $\mathbf{3 k}$ and 3n). Chalcones with electron withdrawing group in the aroyl ring required slightly longer reaction time (entries $\mathbf{3 j}, \mathbf{3} \mathbf{l}$ and $\mathbf{3 m}$ ). However, the mono substituted vinyl ketone (entry 1) gave the Michael adduct at room temperature with excellent yield. The results are in good agreement with earlier reports. ${ }^{19}$

\subsection{Minimum inhibitory concentration (MIC)}

The activity of the 15,3-alkylated indoles was assessed in terms of minimum inhibitory concentraion (MIC) by microdilution assay method against four bacterial strains using 96 well plates. MIC is defined as the maximum dilution of the test compound that inhibits the growth of the microrganism.

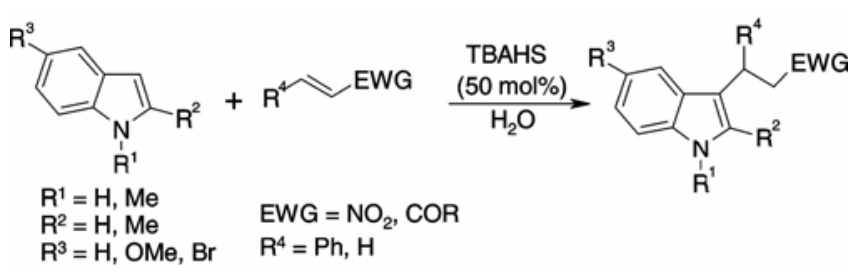

Scheme 1. Michael addition of indoles with various electron deficient olefins. 
Table 1. TBAHS catalysed Michael addition in water.

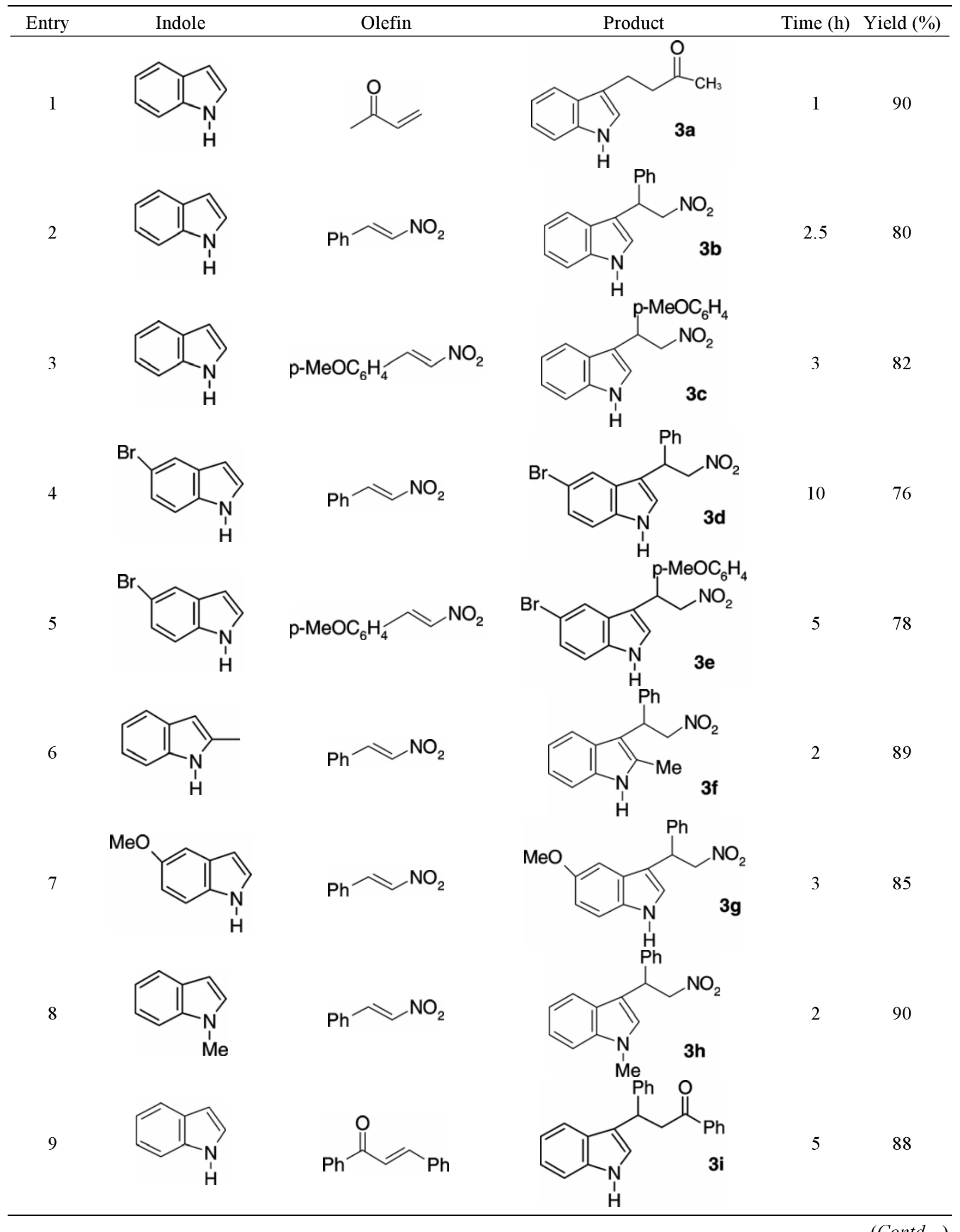


Table 1. (Contd ...)

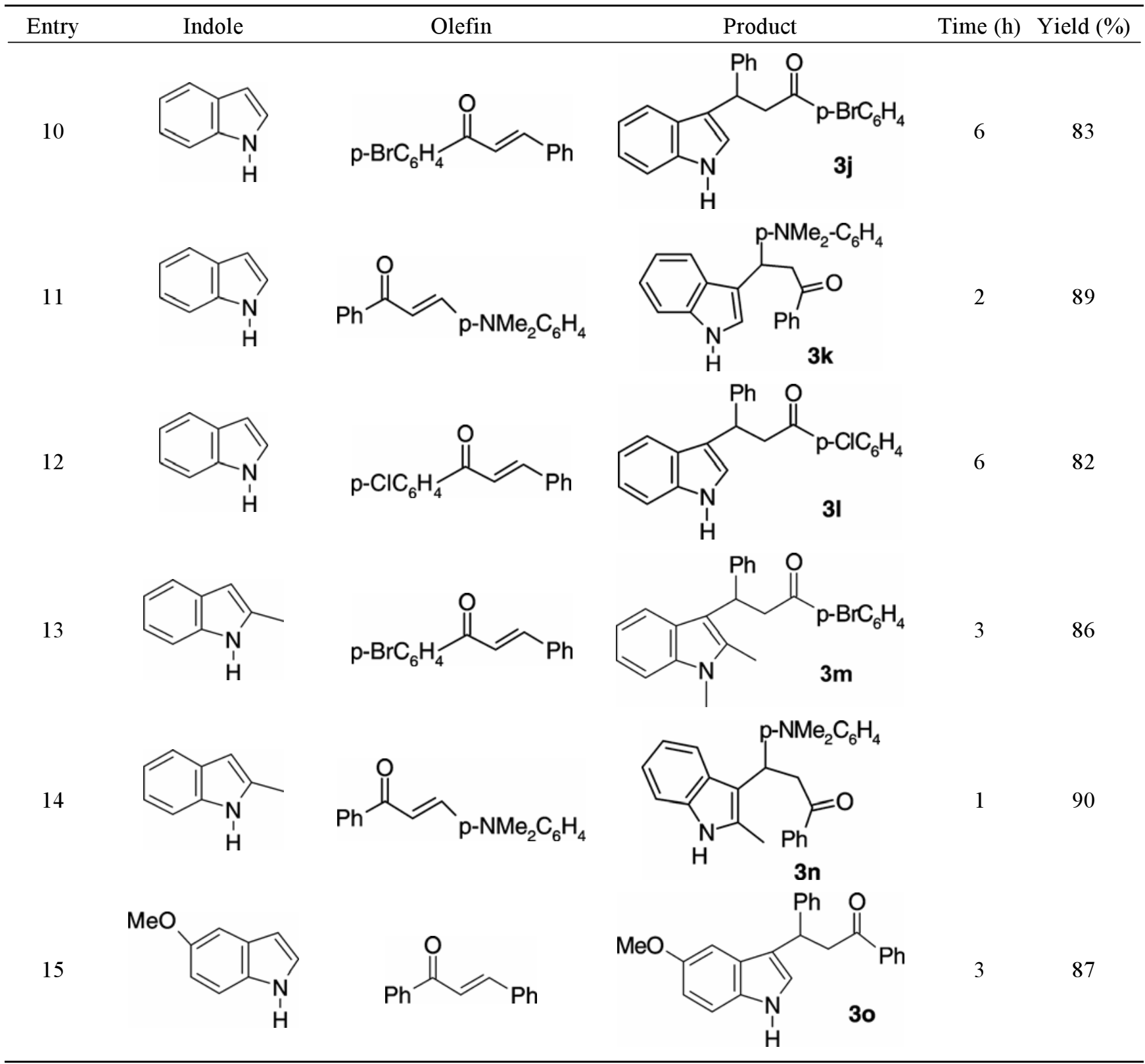

The compounds were dissolved in $10 \%$ DMSO (dimethylsulfoxide) and mixed with Muller-Hinton broth. The plates were incubated initially for $18 \mathrm{~h}$ at $37^{\circ} \mathrm{C}$ and then, $0.01 \%$ resazurin solution was added and incubated for two hours. Resazurin is a redox indicator which remains blue in colour in its oxidized form and when reduced becomes pink coloured intermediate called resorufin. This reaction is irreversible. Pink coloured resorufin is further reduced to colourless dihydroresorufin, but this reaction is irreversible. In the presence of live bacterial cells, which mediate the reduction reaction due to the presence of oxidoreductase enzyme, the blue colour resazurin will turn into pink colour resorufin. ${ }^{20}$ Highest dilution of the compound (growth inhibition) at which the blue colour remains is considered as the MIC of the compound. These compounds are active in the range of $0 \cdot 16-2.76 \mu \mathrm{M}$ concentrations and the results are given in table 2 . Based on the tabulated results, it is concluded that these compounds showed potent inhibitory activity against Gram (-)ve organisms (E. coli NCIM 2931, P. vulgaris NCIM 2813, $P$. aeruginosa NCIM 5029) when compared to the Gram (+)ve organism (S. aureus NCIM 5021). 
Table 2. Antibacterial activity of synthesized 3-alkylated indole derivatives against four bacterial starins.

\begin{tabular}{|c|c|c|c|c|c|}
\hline \multirow[b]{2}{*}{ Series } & \multirow[b]{2}{*}{$\begin{array}{c}\text { Compound } \\
\text { no }\end{array}$} & \multicolumn{4}{|c|}{ Antibacterial activity (minimum inhibitory concentration) in $\mu \mathrm{M}$} \\
\hline & & $\begin{array}{c}\text { E. coli } \\
\text { NCIM } 2931\end{array}$ & $\begin{array}{c}\text { S. aureus } \\
\text { NCIM } 5021\end{array}$ & $\begin{array}{c}\text { P. aeruginosa } \\
\text { NCIM } 5029\end{array}$ & $\begin{array}{c}\text { P. vulgaris } \\
\text { NCIM } 2813\end{array}$ \\
\hline \multirow[t]{8}{*}{ I } & $3 \mathbf{a}$ & $0 \cdot 334$ & $2 \cdot 670$ & $0 \cdot 334$ & 0.334 \\
\hline & 3b & $0 \cdot 939$ & $0 \cdot 469$ & $1 \cdot 878$ & 1.878 \\
\hline & 3c & $0 \cdot 422$ & $1 \cdot 687$ & $0 \cdot 211$ & 0.422 \\
\hline & 3d & $0 \cdot 362$ & $0 \cdot 362$ & $0 \cdot 362$ & 0.362 \\
\hline & $3 \mathbf{e}$ & $0 \cdot 333$ & $0 \cdot 167$ & $0 \cdot 666$ & 0.333 \\
\hline & $3 f$ & 0.223 & 1.784 & $0 \cdot 223$ & 0.223 \\
\hline & $3 g$ & 0.422 & 1.687 & $0 \cdot 211$ & 0.422 \\
\hline & $3 h$ & 0.446 & 1.784 & 0.223 & 0.446 \\
\hline \multirow[t]{7}{*}{ II } & $3 \mathbf{i}$ & 0.768 & 1.537 & $0 \cdot 384$ & 0.384 \\
\hline & $3 \mathbf{j}$ & $0 \cdot 618$ & $1 \cdot 237$ & $1 \cdot 237$ & 1.237 \\
\hline & $3 \mathbf{k}$ & $0 \cdot 654$ & $1 \cdot 307$ & $0 \cdot 163$ & 0.654 \\
\hline & 31 & $0 \cdot 347$ & $1 \cdot 389$ & $0 \cdot 174$ & 0.347 \\
\hline & $3 m$ & 0.598 & $1 \cdot 195$ & 0.598 & 0.590 \\
\hline & $3 n$ & $0 \cdot 327$ & $1 \cdot 307$ & $0 \cdot 163$ & 0.327 \\
\hline & 30 & $0 \cdot 352$ & 1.407 & $0 \cdot 176$ & 0.352 \\
\hline \multicolumn{2}{|c|}{ Erythromycin } & $0 \cdot 0004$ & $0 \cdot 0122$ & $0 \cdot 0004$ & $0 \cdot 0004$ \\
\hline \multicolumn{2}{|c|}{ Norfloxacin } & 0.0027 & 0.0002 & 0.0053 & 0.0053 \\
\hline
\end{tabular}

\section{Conclusion}

We have developed a simple procedure for Michael addition of indoles to a variety of electron deficient olefins using environmental friendly water as the reaction medium. The Michael adducts were obtained in good to excellent yields. This protocol is both operationally simple as the work up involves simple phase separation and economically feasible, as the reaction employs the low cost Tetrabutylmmonium hydrogen sulfate as catalyst and water as reaction medium. The synthesized 3-alkylated indoles showed low MIC values against a broad range of bacteria (both Gram (+)ve and Gram (-)ve) and hence can be possibly considered as antibacterial agents. Earlier studies have proved that the alkaloids containing 3-alkylated indoles as back bone and act as good antimycotic and antibacterial activity. These findings indicate that 3-alkylated indole derivatives are promising broad spectrum antibacterial agents.

\section{Acknowledgements}

One of the authors (MD) thanks University Grants Commission (UGC) and Council of Scientific and Industrial Research (CSIR), New Delhi, India for a senior research fellowship.

\section{References}

1. Chao J L 2005 Chem. Rev. 1053095

2. Li C J and Chang T H 1997 Organic reactions in aqueous media (New York: Wiley)

3. For reviews, see: (a) Sundberg R J 1989 Prog. Heterocycl. Chem. 1111 ; (b) Saxton J E 1986 Nat. Prod. Rep. 3 357; (c) Ezquerra J, Pedregal C and Lamas C 1996 J. Org. Chem. 61 5804; (d) Lobo A M and Prabhakar S $2002 \mathrm{~J}$. Heterocycl. Chem. 39 429; (e) Gribble G W $2000 \mathrm{~J}$. Chem. Soc. Perkin Trans. 1 1045; (f) Xiong W N, Yang C G and Jiang B 2001 Bioorg. Med. Chem. 9 1773; (g) Tokuyama H and Fukuyama T 2001 Kagaku Kogyo 52 416; (h) Toyota M and Ihara M 1998 Nat. Prod. Rep. 15327

4. Fukuyama T and Chen X 1994 J. Am. Chem. Soc. 116 3125

5. (a) Harrington P and Kerr M A 1996 Synlett 1047; (b) Moore R E, Cheuk C and Patterson G M L $1984 J$. Am. Chem. Soc. 1066456

6. Moore R E, Cheuk C and Patterson G M L $1984 J$. Am. Chem. Soc. 1066456

7. (a) Moore R E, Cheuk C, Yang X-Q, Patterson G M L, Bonjouklian R, Smitka T A, Mynderse J, Foster R $\mathrm{S}$, Jones N D, Swartzendruber J K and Deeter J B 1987 J. Org. Chem. 62 3567; (b) Moore R E, Cheuk $\mathrm{C}$ and Patterson G M L $1984 \mathrm{~J}$. Am. Chem. Soc. 106 6456

8. (a) Johnson S, Wilderson J E R, Wenninger M R and Mitchell G S 2001 J. Appl. Physiol. I 91 2703; (b) Monckton J E and McCormick D A 2002 J. Neurophysiol. 87 2124; (c) Vacker C-M Fettier P, Creminon C, Calas A and Hardin-Pouzet H $2002 \mathrm{~J}$. 
Neurosci. 22 1513; (d) Hoey A J, Jackson C M, Pegg G G and Sillence M N 1996 Br. J. Pharmacol. 119 564; (e) Bontchev P R, Pantcheva I N, Bontchev R P, Ivanov D S and Danchev N D 2002 Biometals 15 79; (f) Haubrich D R, Ward S J, Baizman E, Bell M R Bradford J, Ferrari R, Miller M, Perrone M Pierson A K, Saelens J K and Luttinger D $1990 \mathrm{~J}$. Pharmacol. Exp. Ther. 255 511; (g) Ogata A, Yamada Y, Sugiura M, Takayanagi R, Sawada Y and Iga T $2002 \mathrm{~J}$. Pharm. Soc. Jpn. 121793

9. Yadav S, Abraham S, Reddy B V S and Sabitha G 2001 Synthesis 2165

10. Shi M, Cui S-C and Li Q-J 2004 Tetrahedron 606679

11. Zhan Z-P, Yang R-F and Lang K 2005 Tetrahedron Lett. 463859

12. Bartoli G, Bartolacci M, Bosco M, Foglia G, Giuliani A, Marcantoni E, Sambri L and Torregiani E $2003 \mathrm{~J}$. Org. Chem. 684594

13. Mizuno K, Kimura Y and Otsuji Y 1979 Synthesis 688

14. Thomas M, Gesson J-P and Papot S 2007 J. Org. Chem. 724262

15. Tewari N, Dwivedi N and Tripathi R P 2004 Tetrahedron Lett. 459011
16. Bisht S S, Dwivedi N and Tripathi R P 2007 Tetrahedron Lett. 481187

17. Grigoropoulou G, Clark J H, Hall D W and Scott K 2001 Chem. Commun. 547

18. Kumar R S and Perumal P T 2006 J. Heterocyclic Chem. 431383

19. (a) Zhan Z-P, Yang R-F and Lang K 2005 Tetrahedron Lett. 46 3859; (b) An L-T, Zou J-P, Zhang LL and Zhang Y 2007 Tetrahedron Lett. 48 4297; (c) Bandini M, Fagioli M, Melchiorre P, Melloni A and Achille Umani-Ronchi A 2003 Tetrahedron Lett. 44 5843

20. Sandholm M, Ali-Vehmas T, Nyholm K, HonkanenBuzalski T and Louhi M 1991 Failure mechanism in lactationnal therapy of staphylococcal mastitis Flem. Vet 62 (Suppl 1) pp 171-186

21. Sarker S D, Nahar L and Kumarasamy Y 2007 Methods $\mathbf{4 3} 321$

22. Sarkar S D, Nahar L and Kumarasamy Y 2007 Methods 42321

23. McNicholl B P, McGrath J W and Quinn J P 2006 Water Res. 41127

24. Bigalke D L 1984 Dairy Food Sanit. 4189 